\title{
Reliability Analysis on the Injection System by Mapping T-S Fault Trees into Bayesian Networks
}

\author{
Yaxin Liu ${ }^{1,}$, Zijian Zhang ${ }^{1}$ and Ming Zhong ${ }^{1, b^{*}}$ \\ ${ }^{1}$ School of Naval Architecture and Ocean Engineering, Harbin Institute of Technology at Weihai, \\ China, 264209 \\ aliuyaxin@hit.edu.cn, ${ }^{\mathrm{b}}$ zhongming@hit.edu.cn
}

\begin{abstract}
Keywords: reliability analysis, T-S FTA, Bayesian network, injection system, importance, pharmacy robot.

Abstract. A novel reliability analysis technique based on Bayesian network and T-S FTA is proposed in this paper. In the proposed technique, the nodes in Bayesian network can be expressed in terms of fuzzy possibilities and the magnitudes of the failure in the system are expressed in term of fuzzy variables. The paper shows that any T-S fault tree can be directly mapped into a BN and that forward inference techniques on the latter may be used to obtain classic parameters computed from the former (i.e. reliability of Top Event or any sub-system, the importance of Basic Events, etc). Furthermore, by using BN, a general diagnostic analysis (backward inference) can be performed, in which posterior probability is computed. The proposed technique is applied to analyze the reliability of the injection system of chemotherapy pharmacy robot.
\end{abstract}

\section{Introduction}

Chemotherapy pharmacy robot is a complex electromechanical system to achieve preparation of chemotherapy drugs automatically and injection system is one of key sub-systems inside, which is applied to inject solvent from syringe to drug vials. Considering the requirement of seasonable treatment for patients, and the possibility of losing effectiveness for chemotherapy drugs after being dissolved for a long time, the injection should be completed in time. Furthermore, any error in the type or dosage of drugs prepared cannot be allowed. To this end, it is necessary to carry on reliability analysis for the injection system.

Fault tree analysis (FTA) is a visual and effective method to analyze dependable systems [1,2], yet it is difficult for complex system to build and analyze fault tree model, due to the uncertainty of failure probability of components and the uncertainty of principle of failure. T-S fuzzy FTA based on T-S model and fuzzy theory is more robust than conventional fault tree [3]. In this technique, fuzzy variables were used to describe both fuzzy probability and magnitude of the failure, and the traditional logic gate was replaced by T-S gate to describe the association of components. In order to measure the impact of each basic event on the top event, T-S fuzzy importance of multistate system [4] had been defined. However, this technique has two disadvantages: large amount of calculation in forward inference based on the basic event information and lack of capacity for backward inference, which limit its application in practical engineering.

On the other hand, Bayesian network $(\mathrm{BN})$ based on graph and probability theory is a kind of directed graph description for dependable systems [5]. With a bidirectional inference mechanism, it was used as a robust and efficient framework for reasoning with uncertain knowledge [6]. Fault tree can be directly mapped into a BN and that basic inference technique on the latter can be used to obtain classical parameters computed from the former [7]. However, the limitations of conventional fault tree restrict the application of $\mathrm{BN}$.

In this paper, an improving analysis technique for dependable systems based on T-S FTA and BN is proposed, in which T-S fault tree can be mapped into a BN directly to overcome the limitations of conventional fault tree, and bidirectional inference can be used to analyze T-S fault tree quantitatively to overcome the two disadvantages of T-S FTA. The method of modelling Bayesian network based on T-S FTA is derived, and reliability parameters are obtained such as reliability of leaf node or any 
sub-node, importance of root nodes and posterior probability of root nodes. Furthermore, the application of the proposed TS-FTA to the injection system of chemotherapy pharmacy robot is presented as well as the conclusions.

\section{Reliability Analysis Methodology}

Bayesian Network. Bayesian Network contains a directed acyclic graph (DAG) in which discrete variables are assigned to each node and edges represent the causal probabilistic relationship among the nodes. BN also contains several conditional probability tables (CPT) defining probability distribution over the nodes [8].

The topology of the network, a qualitative part, is represented by DAG in which edges connects the nodes from a parent node to a child node. Root nodes are nodes with no parents, while leaf nodes are nodes with no children, and other nodes are sub-nodes. On the other hand, CPT, a quantitative part, contains, for each possible value of the variables associated to a node, all the conditional probabilities with respect to all the combination of values of the variables associated to the parent nodes and prior probabilities are associated with root nodes.

T-S fault tree is defined by a series of T-S gates following IF-THEN rules, taken from the literature [3]. When mapping T-S fault tree into Bayesian network, basic events, sub-events and top events in former can be transformed into root nodes, sub-nodes and leaf nodes, respectively. Based on the logic relationship defined by T-S gates, edges are used to connect each node. Further, CPT is assigned by $\mathrm{T}-\mathrm{S}$ gate rules. The modelling process is as shown in Fig.1.

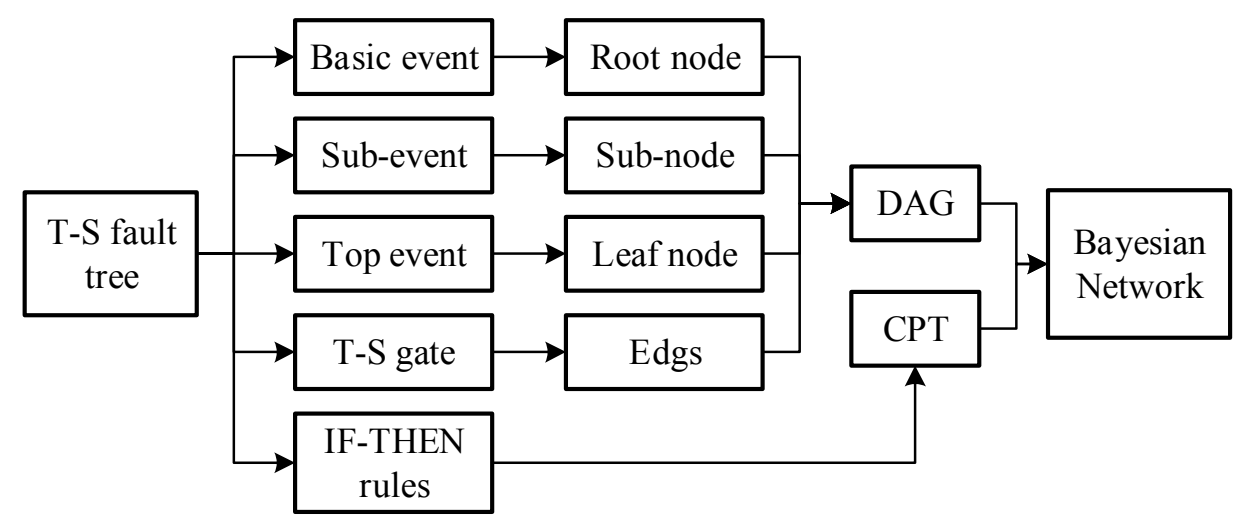

Fig.1 Modelling process of Bayesian network based on T-S fault tree

Node Description. Fuzzy variable $\tilde{P}$ with a linear triangle membership function defined on probability interval $[0,1]$ is used to describe the fuzzy possibilities of failure of nodes, and $\tilde{P}$ is denoted by,

$$
\tilde{P}=\left(p^{l}, p^{m}, p^{r}\right)
$$

where, $p^{m}$ is the center of the support set, whose membership degree is $1, p^{l}$ and $p^{r}$ are respectively the left and the right distribution and $0 \leq p^{l} \leq p^{m} \leq p^{r} \leq 1$. The membership function can be expressed as follows,

$$
\mu_{\tilde{P}}(p)=\left\{\begin{array}{l}
\max \left\{0, \frac{p-p^{l}}{p^{m}-p^{l}}\right\} \quad 0 \leq p \leq p^{m} \\
\max \left\{0, \frac{p^{r}-p}{p^{r}-p^{m}}\right\} \quad p^{m} \leq p \leq 1
\end{array}\right.
$$


Fuzzy variable $F$ with a trapezoidal membership function defined on closed interval $[0,1]$ is used to describe the magnitude of failure of nodes and suppose the magnitude of failures of nodes is either $0,0.5,1$, representing normal, minor failure and complete failure.

Root nodes, sub-nodes and leaf nodes are respectively defined as $x_{j}(j=1,2, \cdots n)$, $y_{i}(i=1,2, \cdots m)$ and $T$, while magnitude of failure of them is defined as $x_{j}^{b_{j}}\left(b_{j}=1,2, \cdots k_{j}\right)$, $y_{i}^{a_{i}}\left(a_{i}=1,2, \cdots k_{i}\right), T_{q}\left(q=1,2, \cdots k_{Q}\right)$, where $0 \leq x_{j}^{b_{j}} \leq 1,0 \leq y_{i}^{a_{i}} \leq 1,0 \leq T_{q} \leq 1$ and $k_{j}, k_{i}, k_{Q}$ represent the quantity of magnitude of failure respectively.

Failure Probability of Leaf Node. In the root nodes, suppose the fuzzy possibilities of the magnitude of the failures are $\tilde{P}\left(x_{j}=x_{j}^{b_{j}}\right)$, then based on the forward analysis of Bayesian network, the fuzzy possibility of the leaf node $T$ whose magnitude of the failure is $T_{q}$ is given by,

$$
\begin{gathered}
\tilde{P}\left(T=T_{q}\right)=\sum_{x_{1}, x_{2}, \cdots, x_{n} ; y_{1}, y_{2}, \cdots, y_{m}} \tilde{P}\left(x_{1}, x_{2}, \cdots, x_{n} ; y_{1}, y_{2}, \cdots, y_{m} ; T=T_{q}\right)=\sum_{\lambda(T)} P\left(T=T_{q} \mid \lambda(T)\right) \times \\
\sum_{\lambda\left(y_{1}\right)} P\left(y_{1} \mid \lambda\left(y_{1}\right)\right) \sum_{\lambda\left(y_{2}\right)} P\left(y_{2} \mid \lambda\left(y_{2}\right)\right) \times \cdots \times \sum_{\lambda\left(y_{m}\right)} P\left(y_{m} \mid \lambda\left(y_{m}\right)\right) \tilde{P}\left(x_{1}=x_{1}^{b_{1}}\right) \tilde{P}\left(x_{2}=x_{2}^{b_{2}}\right) \cdots \tilde{P}\left(x_{n}=x_{n}^{b_{n}}\right)
\end{gathered}
$$

where, $\lambda(T)$ is the failure magnitude set of the parent nodes of leaf node $T, \lambda\left(y_{i}\right)$ is the failure magnitude set of the parent nodes of sub-nodes $y_{i}, P\left(T=T_{q} \mid \lambda(T)\right)$ is conditional probability of the leaf node $T$ whose magnitude of the failure is $T_{q}$ given $\lambda(T)$.

Probability Importance of Root Node. When the fuzzy possibility of $x_{j}$ whose failure magnitude is $x_{j}^{b_{j}}$ is $\tilde{P}\left(x_{j}=x_{j}^{b_{j}}\right)=(1,1,1)$ (certainly happen), suppose the fuzzy possibility of $T=T_{q}$ is $\tilde{P}\left[T=T_{q}, \tilde{P}\left(x_{j}=x_{j}^{b_{j}}\right)=(1,1,1)\right]$. Similarly, when the fuzzy possibility of $x_{j}$ whose failure magnitude is $x_{j}^{b_{j}}$ is $\tilde{P}\left(x_{j}=x_{j}^{b_{j}}\right)=(0,0,0)$, the fuzzy possibility of $T=T_{q}$ is $\tilde{P}\left[T=T_{q}, \tilde{P}\left(x_{j}=x_{j}^{b_{j}}\right)=(0,0,0)\right]$. The probability importance $I_{x_{j}}^{T_{q}}\left(x_{j}^{b_{j}}\right)$ of $\tilde{P}\left(x_{j}=x_{j}^{b_{j}}\right)$ for $T=T_{q}$ is denoted by,

$$
I_{x_{j}}^{T_{q}}\left(x_{j}^{b_{j}}\right)=E\left\{\tilde{P}\left[T=T_{q}, \tilde{P}\left(x_{j}=x_{j}^{b_{j}}\right)=(1,1,1)\right]-\tilde{P}\left[T=T_{q}, \tilde{P}\left(x_{j}=x_{j}^{b_{j}}\right)=(0,0,0)\right]\right\}
$$

where,

$$
E(\tilde{P})=\frac{\int_{0}^{1} p \cdot \mu_{\tilde{P}} \mathrm{~d} p}{\int_{0}^{1} \mu_{\tilde{P}} \mathrm{~d} p}
$$

The effect of each failure magnitude of $x_{j}$ on $T=T_{q}$ was synthesized by applying arithmetic mean to obtain the comprehensive importance of $x_{j}$ on $T=T_{q}$ [4], in which the difference between each failure magnitude was not considered. In this paper, weighted mean is used to obtain the comprehensive importance $I^{T_{q}}\left(x_{j}\right)$ by taking $x_{j}^{b_{j}}$ as the weight. $I^{T_{q}}\left(x_{j}\right)$ is given by,

$$
I^{T_{q}}\left(x_{j}\right)=\frac{\sum_{i_{j}=1}^{k_{j}} x_{j}^{b_{j}} \times I_{x_{j}}^{T_{q}}\left(x_{j}^{b_{j}}\right)}{\sum_{i_{j}=1}^{k_{j}} x_{j}^{b_{j}}}
$$

Posterior Possibility of Root Node. Based on the Bayes formula, the posterior probability $P\left(x_{j}=x_{j}^{b_{j}} \mid T=T_{q}\right)$ of $x_{j}$ whose failure magnitude is $x_{j}^{b_{j}}$ given $T=T_{q}$ is denoted by, 


$$
P\left(x_{j}=x_{j}^{b_{j}} \mid T=T_{q}\right)=E\left[\frac{\tilde{P}\left(x_{j}=x_{j}^{b_{j}}, T=T_{q}\right)}{\tilde{P}\left(T=T_{q}\right)}\right]
$$

where, $\tilde{P}\left(x_{j}=x_{j}^{b_{j}}, T=T_{q}\right)$ is joint probability.

\section{Pharmacy Robot and Injection System}

As shown in Fig.2, the pharmacy robot is an extremely complex system which consists of seven independent function modules under cooperation, such as dosage station, manipulator etc. The Drug injection system, a key sub-system of the pharmacy robot, mainly comprises a dosage station employed to distribute medical solution with syringe accurately and a manipulator applied to transfer drug vials, bags and syringes among different modules.

An example in which a drug vial filled with pulverous Oxaliplatin is injected with $5 \%$ glucose solution will be discussed here to expound working principle of the injection system. As shown in Fig.3, suppose that a syringe filled with solution has been placed into the dosage station at this time. With the rotation of dosage station, syringe needle is made vertical downward and then the drug vial placed underneath it is raised slowly by the manipulator to make itself pierced through. Finally, syringe piston is pressed to the bottom of the syringe by the core rod actuator with glucose solution injected into the vial.

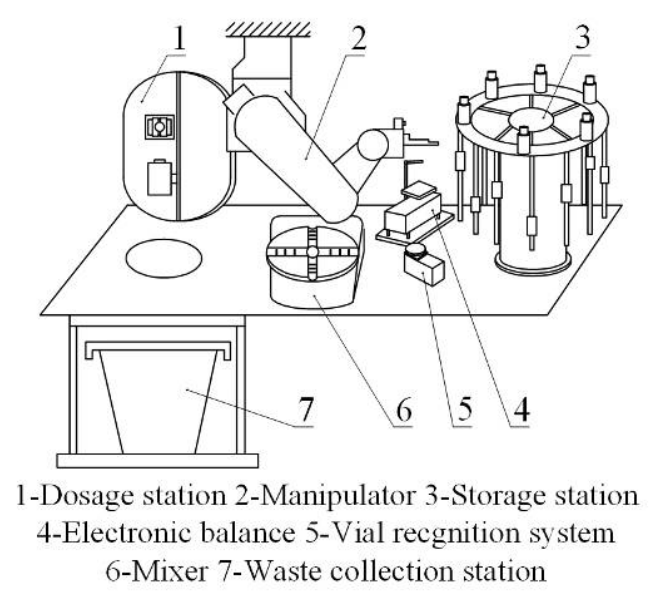

Fig.2 Pharmacy robot system

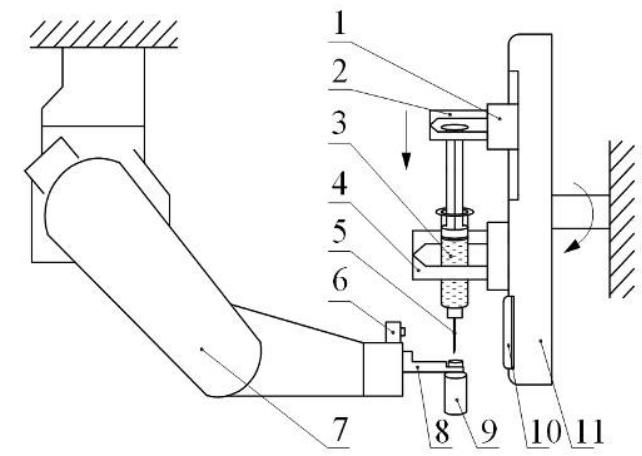

1-Core rod actuator 2-Core rod gripper 3-Syringe 4-Syring jacket gripper 5-Needle 6-Camera 7-Manipulator 8-Robotic gripper 9-Drug vials 10-Blacklight 11-Rotary table

Fig.3 Injection system

It is vital for drug vial to keep its axis consistent with the needle during rising, and to this end, a camera placed towards the approach vector direction of robotic gripper was mounted on the end of arm to detect needle position. Furthermore, a backlight making the contour of needle more visible was mounted on the rotary table. According to the working principle, the injection system involves multiple components with complex relationships and it is necessary to carry on reliability analysis on the injection system of pharmacy robots.

\section{Reliability Analysis of Injection System}

Bayesian Network Modeling. The reliability analysis of the injection system using the T-S fault tree as shown in Fig. 4 is presented here and Bayesian network model of injection system as shown in Fig.5 can be obtained based on T-S fault tree according to the method shown in Fig. 1. The leaf node $T$ is the failure of the system and $y_{1} \sim y_{5}$ are respectively sub-systems as shown in Table 1 .

In the Bayesian network, let the magnitude of failures of $x_{4}, x_{5}$ be either 0,1 , representing normal and failure, while the magnitude of failures of $x_{1} \sim x_{3}, x_{6} \sim x_{7}, y_{1} \sim y_{5}, T$ be either $0,0.5,1$. Refer to the reliability design handbook and expert experience, the conditional probability table of 
sub-node $y_{1}$ is shown in Table 2 and the fuzzy variables of failure probability of root nodes $x_{j}$ whose failure magnitude is 1 can be obtained as shown in Table 3.

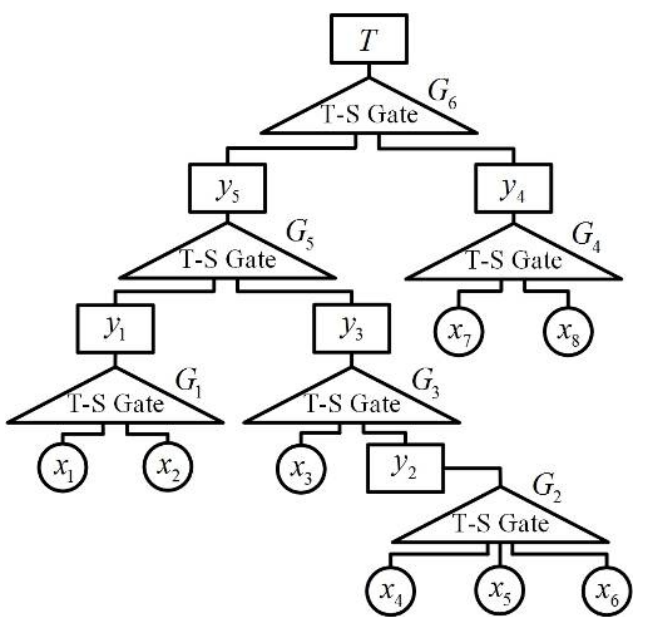

Fig.4 T-S fault tree of injection system

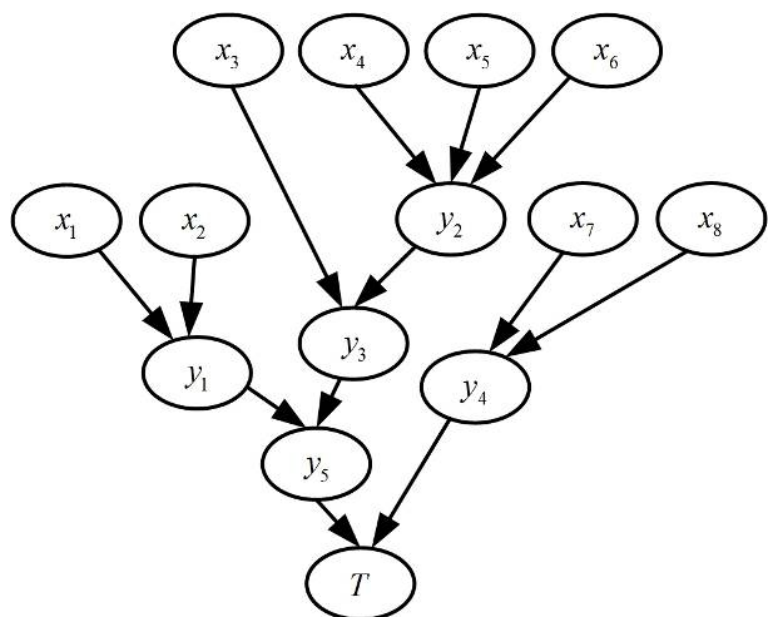

Fig. 5 bayesian network of injection system

Estimation of Failure Possibility of Leaf Node. Suppose the fuzzy possibilities of $x_{1} \sim x_{3}, x_{6} \sim x_{7}$ failing with a magnitude of 0.5 are the same as that with a magnitude of 1 . According to conditional probabilities distribution as shown in Table 2, the fuzzy possibilities of leaf node $T$ failing with magnitudes of $0,0.5$ or 1 can be obtained by expression (3) as follows,

$$
\begin{aligned}
& \tilde{P}(T=0)=\left(999898.14 \times 10^{-6}, 999888.75 \times 10^{-6}, 999879.68 \times 10^{-6}\right) \mathrm{h}^{-1} \\
& \tilde{P}(T=0.5)=\left(22.04 \times 10^{-6}, 23.35 \times 10^{-6}, 24.65 \times 10^{-6}\right) \mathrm{h}^{-1} \\
& \tilde{P}(T=1)=\left(79.82 \times 10^{-6}, 87.75 \times 10^{-6}, 95.67 \times 10^{-6}\right) \mathrm{h}^{-1}
\end{aligned}
$$

Table 1 Sub-systems corresponding to sub-nodes

\begin{tabular}{|c|c|}
\hline Sub Nodes $y_{i}$ & Name of Sub-systems \\
\hline$y_{1}$ & Position system of needle \\
\hline$y_{2}$ & Monocular vision system \\
\hline$y_{3}$ & Position system of vial \\
\hline$y_{4}$ & Position system of core rod \\
\hline$y_{5}$ & Piercing motion sysytem \\
\hline
\end{tabular}

Table 2 Conditional probability table of sub-node $y_{1}$

\begin{tabular}{|c|c|c|c|c|c|}
\hline \multirow{2}{*}{ No. } & \multirow{2}{*}{$x_{1}$} & $x_{2}$ & \multicolumn{3}{|c|}{$y_{1}$} \\
\cline { 4 - 6 } & & & 0 & 0.5 & 1 \\
\hline 1 & 0 & 0 & 1 & 0 & 0 \\
\hline 2 & 0 & 0.5 & 0.1 & 0.5 & 0.4 \\
\hline 3 & 0.5 & 0 & 0.4 & 0.4 & 0.2 \\
\hline 4 & 0.5 & 0.5 & 0.1 & 0.2 & 0.7 \\
\hline $5 \sim 9$ & & - & 0 & 0 & 1 \\
\hline
\end{tabular}

Table 3 Fuzzy variables of failure probability of root nodes

\begin{tabular}{|c|c|c||c|c|c|}
\hline $\begin{array}{c}\text { Root } \\
\text { Nodes } \\
x_{j}\end{array}$ & Components & $\begin{array}{c}\text { Fuzzy Variables of } \\
\text { Failure Probability } \\
\tilde{P}\left(x_{j}=1\right) /\left(10^{-6} / \mathrm{h}\right)\end{array}$ & $\begin{array}{c}\text { Root } \\
\text { Nodes } \\
x_{j}\end{array}$ & Components & $\begin{array}{c}\text { Fuzzy Variables of } \\
\text { Failure Probability } \\
\tilde{P}\left(x_{j}=1\right) /\left(10^{-6} / \mathrm{h}\right)\end{array}$ \\
\hline$x_{1}$ & Syring jacket gripper & $(31.6,32.6,33.6)$ & $x_{5}$ & Camera & $(1.4,2.4,3.4)$ \\
\hline$x_{2}$ & Rotary table & $(15.2,16.2,17.2)$ & $x_{6}$ & Blacklight & $(6.4,7.4,8.4)$ \\
\hline$x_{3}$ & Manipulator & $(0,1.0,2.0)$ & $x_{7}$ & Core rod gripper & $(23.2,24.2,25.2)$ \\
\hline$x_{4}$ & $\begin{array}{c}\text { Image acquisition } \\
\text { card }\end{array}$ & $(7.0,8.0,9.0)$ & $x_{8}$ & Core rod actuator & $(5.46 .47 .4)$ \\
\hline
\end{tabular}

Estimation of Probability Importance. The probability importance of root nodes $x_{j}$ for leaf node failing with magnitudes of 0.5 or 1 can be calculated according to (4) (6), as shown in Table 4.

According to Table 4, when the fuzzy variables of failure probability of root nodes have already been known, the probability importance of $x_{3}, x_{5}, x_{7}, x_{8}$ is larger than others, i.e. $x_{3}, x_{5}, x_{7}, x_{8}$ are 
weak links of the injection system. Therefore, during the period of selecting or processing components, they should be paid more attention to. In addition, adding redundancy design to weak links can also improve the reliability of the system.

Estimation of Posterior Possibility. The posterior possibility of root nodes $x_{j}$ failing with different magnitudes given the evidence that leaf node has already been failed can be calculated according to (7), as shown in Table 5.

According to Table 5, when the leaf node has already been failed, the posterior possibilities of $x_{3}$, $x_{4}, x_{5}, x_{6}$ are larger than others, i.e. $x_{3}, x_{4}, x_{5}, x_{6}$ are weak links of the system. Therefore, they should be checked if they are failed prior in fault diagnosis.

Table 4 Probability importance of kinds of root nodes

\begin{tabular}{|c|c|c|}
\hline $\begin{array}{c}\text { Root Nodes } \\
x_{j}\end{array}$ & $I^{0.5}\left(x_{j}\right)$ & $I^{1}\left(x_{j}\right)$ \\
\hline$x_{1}$ & 0.1440 & 0.3414 \\
\hline$x_{2}$ & 0.1613 & 0.3778 \\
\hline$x_{3}$ & 0.0320 & 0.9086 \\
\hline$x_{4}$ & 0.0480 & 0.2861 \\
\hline$x_{5}$ & 0.0480 & 0.6260 \\
\hline$x_{6}$ & 0.0443 & 0.5395 \\
\hline$x_{7}$ & 0.1867 & 0.7933 \\
\hline$x_{8}$ & 0.2223 & 0.7849 \\
\hline
\end{tabular}

Table 5 Posterior probability of kinds of root nodes

\begin{tabular}{|c|c|c|c|c|}
\hline Root Nodes & \multicolumn{2}{|c|}{$P\left(x_{j}=\mid T=0.5\right)$} & \multicolumn{2}{c|}{$P\left(x_{j}=\mid T=1\right)$} \\
\cline { 2 - 5 }$x_{j}$ & 0.5 & 1 & 0.5 & 1 \\
\hline$x_{1}$ & 0.5519 & 0.6190 & 0.7122 & 0.7999 \\
\hline$x_{2}$ & 0.5093 & 0.5065 & 0.6979 & 0.7214 \\
\hline$x_{3}$ & 0.9520 & 0.9500 & 0.8616 & 0.8647 \\
\hline$x_{4}$ & - & 0.9685 & - & 0.8991 \\
\hline$x_{5}$ & - & 0.9570 & & 0.8901 \\
\hline$x_{6}$ & 0.9637 & 0.9672 & 0.9039 & 0.9258 \\
\hline$x_{7}$ & 0.8972 & 0.6069 & 0.4691 & 0.6401 \\
\hline$x_{8}$ & 0.6617 & 0.6069 & 0.4081 & 0.4372 \\
\hline
\end{tabular}

\section{Conclusions}

A new method for analysis of injection system reliability is developed. The method integrates the T-S FTA and Bayesian network. The main conclusions are as follows: (i) Injection system is a key sub-system, whose reliability is relied on the position precision of needles and drug vials. (ii) Manipulator is a key component which has the greatest impact on the failure possibility of the system. (iii) Manipulator, image acquisition card should be checked if they are failed prior in fault diagnosis.

\section{References}

[1] W.S. Lee, D.L. Grosh, F.A. Tillman, et al: IEEE Transactions on Vol. 34 (1985), pp.194-203

[2] A. Volkanovski, M. Čepin, B. Mavko: Reliability Engineering \& System Safety Vol. 94 (2009) pp. 1116-1127

[3] H Song, H.Y. Zhang, C.W. Chan: Soft Comput Vol.13 (2009) pp. 31-40

[4] C.Y. Yao, Y.Y. Zhang, D.N. Chen, et al: Journal of Mechanical Engineering Vol.47 (2011) pp. 163-169 (In Chinese)

[5] H. Langseth, L. Portinale: Reliability Engineering and System Safety Vol.92 (2007) pp. 92-108

[6] A.G. Wilson, A.V. Huzurbazar: Reliability Engineering and System Safety Vol.92 (2007) pp. 1413- 1420

[7] A. Bobbio, L. Portinale, M. Minichino, et al: Reliability Engineering \& System Safety Vol.71 (2001) pp. 249-260

[8] N Khakzad, F. Khan, P. Amyotte: Reliability Engineering \& System Safety Vol.96 (2011) pp. 925-932 